\title{
Performance Evaluation of Re-Routing based Hybrid ACO-PSO based Routing Algorithm for MANETS
}

\author{
Harkirat Kaur \\ M.Tech Scholar \\ Department of CSE \\ Amritsar College of Engineering \\ and Technology, Amritsar , India
}

\author{
Shivani Sharma \\ Associate Professor \\ Department of CSE \\ Amritsar College of Engineering \\ and Technology, Amritsar , India
}

\begin{abstract}
Wireless ad-hoc networks is a parent breed to Mobile ad-hoc networks, comprising of many mobile nodes capable of dynamically exchanging data amongst nodes without demanding a centralized infrastructure. Re-routing based Hybrid ACO-PSO based routing algorithm for MANETs have been discussed in this paper and is studied for evaluating its performance. This algorithm is applicable to multi-hop ad-hoc networks with the aim of upgrading the performance of the existing protocol for mobile ad hoc network. The Re-routing based Hybrid strategy is developed and deployed using MATLAB 2013a and the toolbox used is data analysis toolbox. The performance is evaluated by comparing the technique that already exists to the one that is proposed in this paper. The results have shown that the new techniques outperforms the previous technique.
\end{abstract}

\section{Keywords}

MANET, Hybrid ACO-PSO, Ant colony optimization, particle swarm optimization

\section{INTRODUCTION}

MANET is an accumulation of autonomous cell or nodes that can interact with each through the medium of radio waves. The nodes of cells which are adjacent to each other can communicate directly but the others have to go through intermediate nodes to transfer and transmit their packets. Each node has a software that makes the node capable of collective communication.

\subsection{MANETs Features}

1) Allocated operation: The network is fully distributed , thus it lacks and do not require any type of central control system. All the nodes which are a part of MANET are supposed to communicate collectively and interact independently with each other. and every node tends to be a relay when need arises, like , for applying operations such as data packets routing, and network security.

2) Adjustable hop course-plotting: When a node needs to send packets to some other node or nodes within its conversation range, it has to transmit packet to that node which has to go through several intermediate nodes before it can reach the target node.

3) Autonomous cells: In MANET, each cell node is selfgoverning and self-reliant. In addition to that, each cell can play as router.

4) Powerful topology: Nodes are free to move randomly with independent speeds. This results in unpredictable changes in topology. Due to this reason the MANETs need dynamic routing protocols.

5) Light-weight terminals: Usually, MANET nodes are terminals with less computing power and less memory. Other features about the nodes are also light weight only.

6) Shared Physical Medium: As MANETs use radio waves for communication to be wireless, this medium is accessible to any node that has appropriate resources and equipment required for the purpose .

\subsection{Advantages of MANETs}

Ad-Hoc networks offer many benefits, some of which are listed below :

- MANETs provide information access irrespective of the physical location. So the network is free from geographic constraints.

- Freedom from the central governance of the network.

- $\quad$ Nodes capable of working as routers when required.

- More Scalable-Its very easy and convenient to add more nodes to the network

- Very flexible as compared to equivalent wired alternative.

- $\quad$ Provides more flexibility .

- This network can be configured from anywhere at nay period of time.

- Costs less than equivalent wired network

\subsection{MANETs Problems}

1) Bandwidth constraints: Wireless networks ought to have less capacity than wired networks. Thus the network is unable to harness maximum bandwidth that radio waves based network can provide.

2) Powerful topology: As nodes can leave or join the network at any time as they please , this forms trust problems within the nodes.

3) Redirecting Overhead: With the nodes dynamically and unpredictably changing their positions in the network, stagnant routes are generated. If a route is generated and any node which is a part of that rote leaves the network then that route becomes a useless and a new route needs be generated. This causes many overheads.

4) Transmission errors and packet loss: node mobility leading to frequent path failures, packet collision, hidden 
terminals and many other problems like this causes high packet loss in wireless networks.

5) Power constraints: As the terminals used in MANETs are light weight thus they have power sources with limited power capacity.

6) Security issues: Wireless networks are very vulnerable to security attacks . the reasons being the wireless medium that is prone to data theft and tempering and the whole working is based on interaction of nodes so malicious node can harm the network.

\subsection{MANETs Applications}

1) Military battlefield: The military must require a commonplace network in order to keep the army up to date with the battlefield events. Thus to enable data interchange among its solders and all the army vehicles and send all this data to the headquarters the military can take advantage of MANETs.

2) Team operations: In business environments where most of the projects are handled by teams rather than individuals, these networks come handy to keep the team in touch and connected.

3) Local communication: Mobile ad-hoc networks can be used to configure small networks comprising of, for instance, all the devices in a small building or a school .

4) Short range networks: MANETs can be used to build small networks to connect just a few devices maybe owned by just one individual. Bluetooth is one example of such network.

5) Emergency relief operations: Mobile ad-hoc networks can be of great help during disaster management operations. In situations like earthquakes and flood relief operations when the whole conventional communication infrastructure is damaged, only ad-hoc networks can keep the rescue team and volunteers connected.

\section{RELATED WORK}

[1] This paper discusses the routing protocols that handle node failure by exploring the network redundancy through multipath routing. The issue is sorted by applying end-to-end estimation-based algorithm. This algorithm uses two techniques: one for selecting the route and other for estimating the route. The process of selecting the route is improved by applying confirmation and dropping procedures. [4] The algorithm proposed in this paper optimizes many parameters to resolve the issue Traffic Engineering Multicast. The results from simulation proves that the algorithm proposed in this paper is better than the conventional algorithm that generates multicast tree in many sizes. [5] The deployment of fault tolerant location management named FTLM has been discussed in the paper. All source nodes are dependent in some other nodes for verifying locations. Faults are handled by constantly updating the servers that hold the value of locations. These servers are also only nodes that assist the source nodes. The overheads that occur during the management of storing various locations are also dealt with the help of aggregation. Results have proven that the algorithm used in this paper is more scalable than its competitors using location management. [8] Source initiated Mesh along with soft-state based QoS Probabilistic multicast routing protocol is discussed in this paper with reference to MANETs. In ant colony optimization protocol nodes are depicted as ants. Protocol makes an ant choose the best route with help of other ants' choices. Proposed protocol uses probability based forwarding for making routing decisions. [15] Upgrading security and reducing the count of malicious and misbehaving terminals or cells is a major concern for MANETs , so many researchers are trying to solve these problems. Misconduct of many nodes can lead to worsening the performance of the network.

These nodes use parameter values of the network and temper with those values to downgrade the network performance degrading throughput and increasing delays. To cope with this problem, the technique that has been put to work is called particle swarm optimization. This technique helps to improve the values of the parameters that have been degraded by misconducting nodes. [16] The consideration that wireless ad-hoc networks face problems like routing, usage of the amount of energy, deployment of nodes, is made by the author in this paper. Various researchers have put in their valuable time and energy to sort out the problem discussed above. Lately, these researchers are putting their trust in the technique called swarm optimization. The paper studies many routing protocols that attempt to deal with the same problem. [17] A fundamental set of rules has been established by the Agent-based routing. All the nodes in the network are ought to be guided by this particular set of rules. The interaction between the nodes also take place using this set of rules. The behavior of a group of animals and even some insects is what has inspired the miraculous idea of the concept of Swarm intelligence. The paper talks about different swarm intelligence techniques and how they are or are not beneficial. It also says how performance can be boosted using this algorithm for the network. [20] The paper has described the detection and maintenance of optimal path between the nodes as its ultimate aim. The detection also includes detecting the selfish nodes in the network. The performance of a routing protocol is realized to be boosted by using this. The search for an optimal route reaches its aim by deploying DSR protocol The successful detection of misbehaving nodes is the mere evidence of the success of the protocol itself. It also makes use of ant colony optimization approach.

\section{PARTICLE SWARM OPTIMIZATION (PSO)}

PSO confides in natural biological behavior of animals such as a swarm of birds along with technological aspects of computer science. In improvement, PSO deploys the approach that birds in a swarm use to move forward to benefit the whole swarm and find the best position for every individual bird to benefit itself too at the same time. So, we can say that PSO is essentially based on the following concepts.

\subsection{Cultural Qualities}

Just as humans learn not from themselves but also from the society and our perception about everything and actions we take is largely based on what we hav learned and picked up from the society. In the very similar way, In PSO the birds in the swarm relies on the social behavior of the swarm as a whole to decide in which direction and with what velocity they have to fly. So each individual bird flies according to the global best position to advance the swarm in the intended direction.

\subsection{Principles of Swarm Intelligence}

As PSO is a branch of Swarm intelligence, thus is follows principles of swarm intelligence .

The swarm as a whole and each individual in the swarm is capable of carrying out space time computations. The elements (birds) of the swarm also care about the quality 
factors the swarm maintains. Although every particle of the swarm is self governing, still the swarm has to be stable all the time. This means that the particles should not change behavior as the environment changes. The particles should only act flexible when the change in computation is worth the change in behavior, like in situations when the behavioral changes can lead to better performance of the swarm.

\section{ANT COLONY OPTIMIZATION (ACO)}

An able archetype that can assist in modelling meta-heuristic algorithms for problems involving combinations is Ant Colony Optimization (ACO) . ACO is based on the idea that if you deploy enough many number of simple self-reliant agents to work on finding a solution to some problem involving complicated combinational computational problems then good solutions can be reached.as every other socially behaving element, artificial ants also need a common medium to interact with each other. Pheromone deposited by ants act as that common interaction platform for ants. Ants keep on finding new solutions and good solutions are kept on strengthening by more pheromone deposited on the path with good solution. This is how ants find best optimized solution with the help of ACO algorithm

\section{PROPOSED METHODOLOGY}

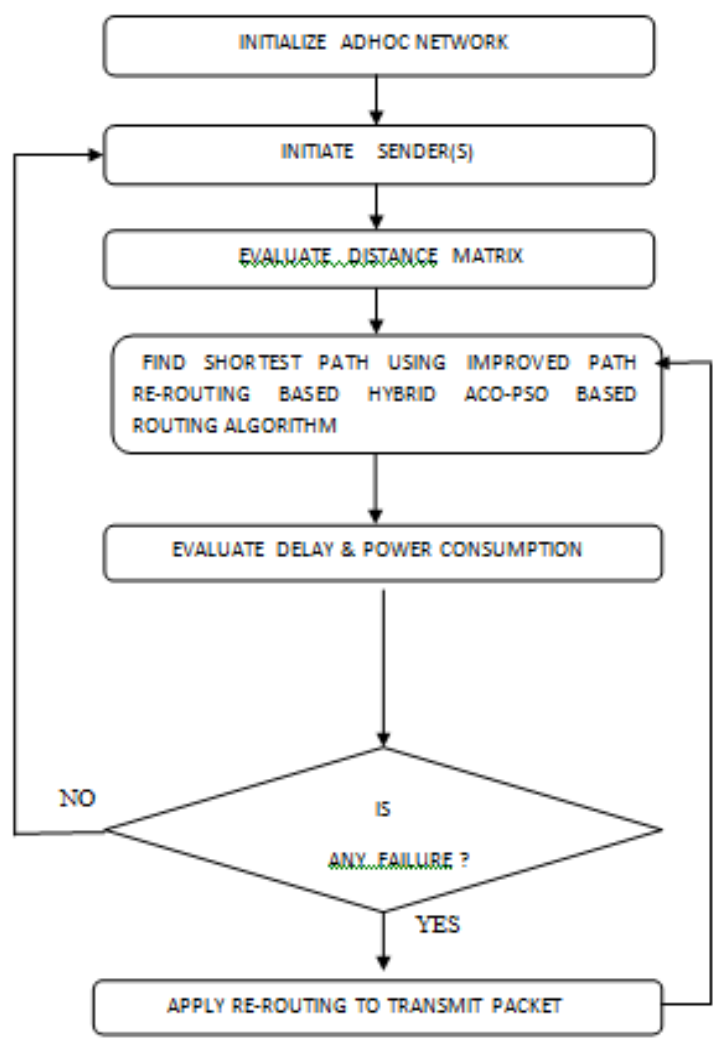

Figure 1: Flowchart of Proposed Method

Step 1: The initialization of Ad-hoc network circle along with its parameter values.

Step 2: Initialization of Sender(s) and multicasting of sender data to its defined nodes.

Step 3: Calculation of the Distance Matrix.
Step 4: Application of re-routing using re-routing based hybrid ACO-PSO based routing technique improved way rerouting dependent hybrid ACO-PSO.

Step 5: Evaluation of power consumption and end-to-end delay

Step 6: When failure is encountered, use re-routing to continue to transmit the packet, else go to step2.

\section{RESULTS AND DISCUSSION \\ 6.1.End to End Delay}

The amount of time that it takes for a packet to reach from its source to the destination is called end-to-end delay. The ratio of the actual number of the packets received at the destination to the actual number of the packets sent from the source is the throughput

Table 1: End To End Delay Values

\begin{tabular}{|l|l|l|}
\hline $\begin{array}{l}\text { Number of } \\
\text { nodes }\end{array}$ & $\begin{array}{l}\text { End to end delay } \\
\text { (existing) }\end{array}$ & $\begin{array}{l}\text { End to end delay } \\
\text { (proposed) }\end{array}$ \\
\hline 100 & 1 & 1 \\
\hline 150 & 3 & 2 \\
\hline 200 & 5 & 3 \\
\hline 250 & 3 & 2 \\
\hline 300 & 1 & 1 \\
\hline 350 & 3 & 2 \\
\hline 400 & 5 & 3 \\
\hline
\end{tabular}

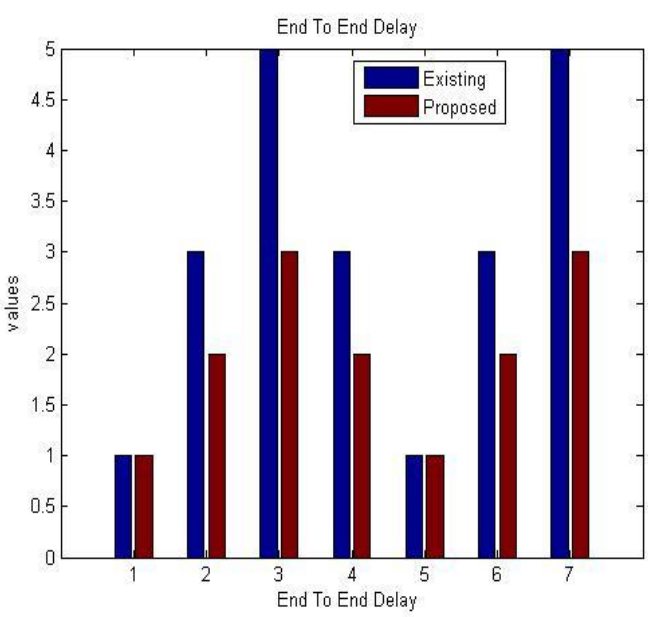

Fig. 2: End To End Delay Graph

\subsection{Power Consumption}

The amount of electrical energy actually used by the network is called the power consumption of that network. The less power the network uses the more efficient it is considered to be and vice versa.

Table 2: Power Consumption Values

\begin{tabular}{|l|l|l|}
\hline Number of nodes & $\begin{array}{l}\text { Power } \\
\text { consumption } \\
\text { (existing) }\end{array}$ & $\begin{array}{l}\text { Power consumption } \\
\text { (proposed) }\end{array}$ \\
\hline 100 & 6 & 4 \\
\hline 150 & 8 & 7 \\
\hline 200 & 8 & 7 \\
\hline 250 & 10 & 8 \\
\hline 300 & 10 & 8 \\
\hline 350 & 8 & 5 \\
\hline 400 & 8 & 5 \\
\hline
\end{tabular}




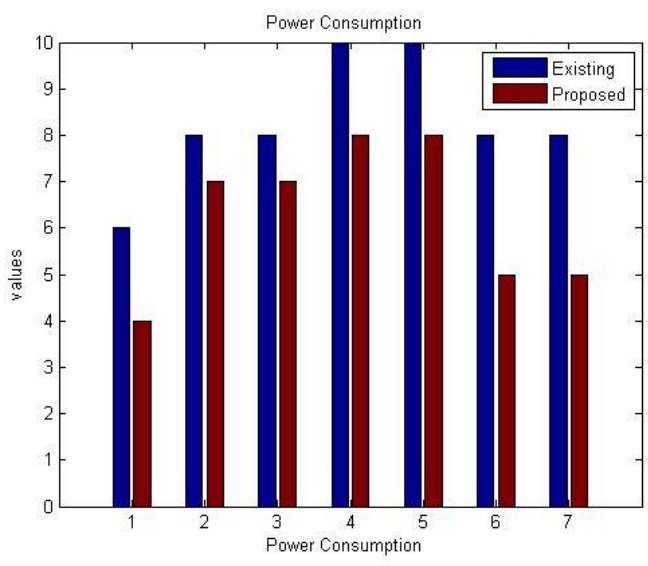

Fig. 3: Power Consumption Graph

\section{CONCLUSION}

With the view of outperforming an overcoming the limitations of earlier work, a new and improved technique is proposed. The proposed technique sorts out the problem of failure of nodes during packet transmission.

Fault tolerance is achieved by the use of path re-routing Rerouting will be done over the node whose successive node has failed. The enforcement of this prproposed technique leads to better values of end -o-end delay and power consumption. The proposed technique is designed and implemented using MATLAB 2013a and the tool used is data analysis toolbox. The proposed technique is also compared with the existing technique to prove that the new technique has a better performance.

This work is centered around handling and detecting the faults during transmission of data in MANETs, also, the application of clustering in order to enhance the power consumption rate is neglected. So, in near future we will enforce various other kinds of techniques and approaches to improve the rate of power consumption.

\section{REFERENCES}

[1] Yuan Xue and Klara Nahrstedt : Fault Tolerant Routing in Mobile Ad Hoc Networks . 0-7803-7700-1/03/\$17.00 (C) 2003 IEEE

[2] Breed Gary. Wireless ad hoc networks: basic concepts. High Freq Electron 2007:44-6.

[3] Munaretto Anelise, Fonseca Mauro. Routing and quality of service support for mobile ad hoc networks. Comput Networks 2007;51(11):3142-56.

[4] Sabari.A and Dr.K.Duraiswamy : Ant Based Multicast Routing Algorithm with Multiple Constraints for Mobile Adhoc Networks . 2008 International Conference on Security Technology

[5] Karim khazaei, Sadegh Mohammadi and Akbar Momeni : A Fault Tolerant Location Management for MANET . 978-1-4244-4565-3/09/\$25.00 @2009 IEEE

[6] Amri Huda Al, Abolhasan Mehran, Wysocki Tadeusz. Scalability of MANET routing protocols for heterogeneous and homogenous networks. ComputElectr Eng 2010;36(4):752-65.

[7] Ali Zulfiqar, Shahzad. Critical analysis of swarm intelligence based routing protocols in ad-hoc and sensor wireless networks. In: International conference on computer networks and information technology; 2011. p. 287-292.

[8] P.Deepalakshmi, Dr.S.Radhakrishnan : SOURCEINITIATED QOS MULTICASTING SCHEME FOR MANETS USING ACO. 978-1-61284-764-1/11/\$26.00 (C)2011 IEEE

[9] Pi Shangchao, Sun Baolin. Fuzzy controllers based multipath routing algorithm in MANET routing. In: International conference on applied physics and industrial engineering. Part B, vol. 24; 2012. p. 1178-85.

[10] Thanushkodi, Deeba K. Hybrid intelligent algorithm [improved particle swarm optimization (PSO) with ant colony optimization (ACO)] formultiprocessor job scheduling. Sci Res Essays 2012;7(20):1935-53.

[11] Aarti, Dr. S. S. Tyagi. A Study of MANET: Characteristics, Challenges, Applicationand Security Attacks. IJARCSSE Volume 3, Issue 5, May 2013

[12] Cheng Hui, Yang Shengxiang, Cao Jiannong. Dynamic genetic algorithms for the dynamic load balanced clustering problem in mobile ad hoc networks. Expert Syst Appl 2013;40(4):1381-92.

[13] Ejaz Waleed, Manzoor Kamran, Kim Hyung Joo, Jang Byung Tae, Jin Gwang-Ja, Kim Hyung Seok. Two-state routing protocol for maritime multi-hop Wireless networks. Comput Electr Eng 2013;39(6):1854

[14] Issac Woungang, Sanjay Kumar Dhurendher, Mohammad S. Obaidat, Alexander Ferwom , Waqas Shah: An ant-swarm inspired energy efficient ad-hoc on demand routing protocol for mobile ad-hoc networks. 978-1-4673-3122-7/13/\$31.00 @2013 IEEE

[15] R. Kalairasi, Dr. Shridharan: Performance improvement of mobile ad hoc network using particle swarm optimization.. Journal of Computational Information Systems 9: 11 (2013) 4213-4221

[16] Sudarshan D Shirkande,Rambabu A Vatti: ACO based routing algorithms for adhoc network(WSN,MANETs): A Survey. 2013 International Conference on communication systems and network topology

[17] Alexandros Giagkos, Myra S. Wilson : BeeIP - A Swarm Intelligence based routing for wireless ad hoc networks .Information Sciences 265 (2014) 23-35

[18] B. Nancharaiah , B. Chandra Mohan :The performance of hybrid routing intelligent algorithm in a mobile ad-hoc network. Computers and Electrical Engineering 40 (2014) 1255-1264

[19] Mahima Chitkara, Mohd. Waseem Ahmad : Review on MANET: Characteristics, Challenges, Imperatives, and Routing protocols. IJCSMC, Vol. 3, Issue. 2, February 2014, pg.432- 437

[20] Tarun Varshney, Aishwary Katiyar, Pankaj Sharma :Performance improvement of MANET under DSR protocol using swarm optimization. 978-1-4799-29009/14/\$31.00 @2014 IEEE

[21] Vallikannu R , A. George , S.K. Srivatsa: Autonomous localization based energy saving mechanism in indoor MANETs using ACO .1570-8667/@ 2014 Elsevier B. V 